\title{
A joint effort to bring together global, regional modeling and proxy communities
}

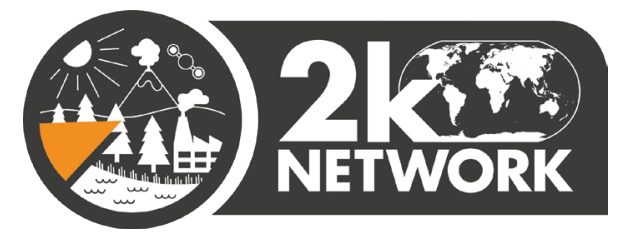

\author{
Juan José Gómez-Navarro', P. Ludwig ${ }^{2}$, N. Zeiher ${ }^{3}$, S. Talento ${ }^{4}$, U. Parveen ${ }^{5}$ and S. Wagner ${ }^{6}$
}

2nd PALEOLINK workshop, Murcia, Spain, 6-8 February 2019

Past climate changes and variations are assessed with proxy reconstructions based on various archives and climate modeling approaches. However, combining both proxy and modeling approaches still includes profound temporal- and spatial-scale gaps. Empirical climate reconstructions are most skillful on a local-to-regional scale covering time periods up to millennia and more, albeit they exhibit a coarse temporal resolution. In contrast, results from comprehensive General Circulation Models (GCM) or Earth system models, which have high temporal resolution, are only representative on regional- to large-scale spatial scales. Thus, innovative and integrated efforts are necessary to bridge the gap between the scales and to bring data and models to a common basis for comparison of past climatic and environmental changes. Therefore, Regional Climate Models (RCM) may be helpful to overcome this spatial and temporal mismatch, but are currently seldom used in the paleo perspective (Fig. 1).

To address these issues, leaders of the PAGES 2k Network project PALEOLINK organized a workshop in the scenic town of Murcia, as a follow up to the PALEOLINK kick-off meeting at the European

Geosciences Union (EGU) General Assembly in Vienna in April 2018. The workshop brought together 22 scientists from different countries - most of them early-career scientists working in the fields of global and regional climate modeling, as well as proxy reconstruction based on different archives and statistical techniques.

The workshop consisted of two sections: a series of oral talks with participants presenting their work and fields of interest, followed by sufficient time for questions, and a series of breakout groups running in parallel.

During the first section, talks were organized around four main topics, ranging from climate reconstructions, using (regional) climate and forward modeling, to modeldata integration. In the second section, several breakout groups were created to address specific open issues and future directions applicable to the entire working group. These groups were not previously defined, but were proposed in situ based on the previous discussions, with the aim to condense ideas stemming and emerging from the preceding talks.

In the first round, four topics were addressed including i) statistical reconstruction methods of hydroclimate variables, ii) identification of variables/regions where the added value in regional paleoclimatic model
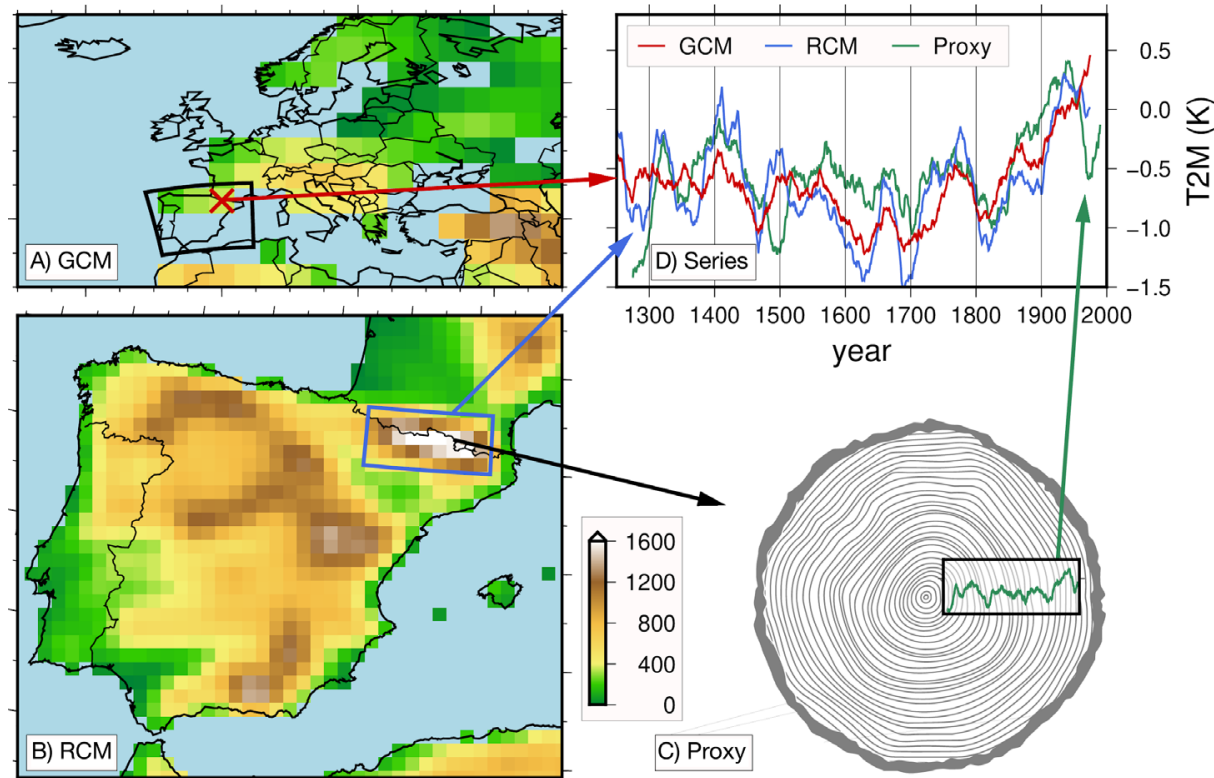

simulations is most noticeable, iii) regional oceanographic models, and iv) regional glacial and interglacial concepts and models. In the second round, workshop participants were encouraged to change groups, thereby sharing their experiences and expertise in order to co-develop strategies and synergetic structures between the different groups. The workshop concluded with summarizing the main results and defining strategies for workshop products related to scientific papers and research initiatives led by enthusiastic group leaders, and coordinating the goals and tasks within the various groups.

An open follow-up meeting took place in a splinter meeting at EGU 2019, where attendants and interested new colleagues in the field of paleoclimatic and paleoenvironmental research had the opportunity to be involved in post-workshop activities. In the future, we plan to aim for additional meetings in the form of online webinars and in-person meetings at larger conferences. In particular, the PALEOLINK leaders are co-conveners of a session at the 20th INQUA congress in July 2019 in Dublin. The group is completely open to input and active participation from the paleoclimatic and paleoenvironmental community interested in addressing issues in the context of the link between the different paleoclimatic spatial and temporal scales.

\section{AFFILIATIONS}

'Department of Physics, University of Murcia, Spain ${ }^{2}$ Institute of Meteorology and Climate Research, Karlsruhe Institute of Technology, Germany ${ }^{3}$ Department of Geography, Friedrich Alexander University, Erlangen, Germany

${ }^{4}$ Department of Geography, Justus Liebig University, Giessen, Germany

${ }^{5}$ Center for the Study of Regional Development Jawaharlal Nehru University, New Delhi, India Institute of Coastal Research, Helmholtz-Zentrum Geesthacht, Germany

CONTACT

Juan José Gómez-Navarro: jjgomeznavarro@um.es

REFERENCES

Ludwig P et al. (2018) Ann. N.Y. Acad. Sci. 1436: 54-69

Figure 1: Schematic of GCM/RCM-proxy data comparison for temperature in the Pyrenees: (A) part of the GCM model domain (orography shaded). Black box marks RCM domain, red cross marks grid point for time series data in (D); (B) RCM model domain (orography shaded). Blue box marks area averaged over the Pyrenees for RCM data in (D), black arrow illustrates location of (C) tree rings used as proxy. (D) Synopsis of GCM, RCM, and proxy data time series. Figure taken from Ludwig et al. (2018) with permission. 